\title{
The value of contrast-enhanced ultrasound in determining the location of sentinel lymph nodes in breast cancer
}

Jun Luo ${ }^{1+}$, Liting Feng ${ }^{1+}$, Qing Zhou ${ }^{1 *}$, Qin Chen ${ }^{1 *}$, Jinping Liu², Chihua Wu², Jing Luo ${ }^{2}$, Jie Chen ${ }^{2}, H_{a o} W^{1}$ and Wanyue Deng ${ }^{1}$

\begin{abstract}
Background: This study aimed to explore the sentinel lymph node (SLN) identification rate in breast cancer by subcutaneous and intradermal injection of ultrasound contrast agent in the mammary areola region, compared to the results achieved with methylene blue (MB).

Methods: A total of 390 breast cancer patients with planned sentinel lymph node biopsy from our breast surgery department from July 2017 to February 2019 were enrolled. All patients were subjected to preoperative contrastenhanced ultrasound (CEUS), that involved an intracutaneous injection of $1 \mathrm{~mL}$ ultrasonic contrast agent (UCA) at 3 and 6 o 'clock, as well as a subcutaneous injection of $1 \mathrm{~mL}$ UCA at 9 and 12 o'clock. The enhanced lymph nodes along the enhanced lymphatic vessels from the mammary areola were traced. The number of enhanced lymph nodes were recorded, and an ultrasound-guided injection of 1:10 diluted carbon nanoparticles were used to mark all first site enhanced lymph nodes (i.e., SLNs). An intraoperative dye method (MB) was used to track the SLNs and the results were compared with the CEUS findings.

Results: Among the 390 cases of breast cancer, enhanced SLNs were observed in 373 patients after an injection of UCA with an identification rate of $95.64 \%$ (373/390), compared to the identification rate of $92.05 \%$ (359/390) using the intraoperative MB. The difference between the two methods was statistically significant $(P=0.016)$. And among the 390 patients, a total of 808 enhanced lymph nodes were traced by preoperative CEUS, with a median of $2(1,3)$. A total of 971 blue-stained lymph nodes were traced using the intraoperative $M B$, with a median of $2(2,3)$, indicating a statistically significant difference $(p<0.001)$.

Conclusions: Intradermal and subcutaneous injections of UCA in the mammary areola region may have clinical application value for the identification and localization of SLNs in breast cancer patients. The identification rate is higher than that of blue dye method, which can be used as a new tracer of sentinel lymph node biopsy and complement other staining methods to improve the success rate.
\end{abstract}

Keywords: Contrast-enhanced ultrasound (CEUS), Breast cancer, Sentinel lymph node (SLN)

\footnotetext{
* Correspondence: uszhouqing@hotmail.com; 1718686103@qq.com

†Jun Luo and Liting Feng contributed equally to this work.

'Department of Ultrasound, Sichuan Provincial People's Hospital, University

of Electronic Science and Technology of China, 32\# W.Sec 2,1st Ring Rd,

610072 Chengdu, China

Full list of author information is available at the end of the article
}

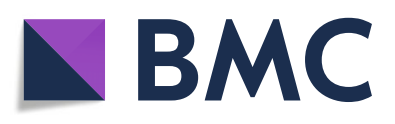

(- The Author(s). 2021 Open Access This article is licensed under a Creative Commons Attribution 4.0 International License, which permits use, sharing, adaptation, distribution and reproduction in any medium or format, as long as you give appropriate credit to the original author(s) and the source, provide a link to the Creative Commons licence, and indicate if changes were made. The images or other third party material in this article are included in the article's Creative Commons licence, unless indicated otherwise in a credit line to the material. If material is not included in the article's Creative Commons licence and your intended use is not permitted by statutory regulation or exceeds the permitted use, you will need to obtain permission directly from the copyright holder. To view a copy of this licence, visit http://creativecommons.org/licenses/by/4.0/. The Creative Commons Public Domain Dedication waiver (http://creativecommons.org/publicdomain/zero/1.0/) applies to the data made available in this article, unless otherwise stated in a credit line to the data. 


\section{Background}

Breast cancer is the most common malignant tumor with the highest morbidity in women; however, breast cancer has a relatively good prognosis and prolonged survival time due to the development of recent treatment methods, such as radiotherapy, chemotherapy, endocrine therapy, and molecular targeted therapy [1]. Despite these methods, surgery remains the primary method of breast cancer treatment. Since the lymph nodes of the ipsilateral axilla drain the majority of the breast lymphatic drainage systems (>75\%), the axillary lymph node (ALN) status is an important factor that affects the prognosis of breast cancer patients. It is known that malignant spread to ALN increases the 10-year recurrence rate from $20 \%-30-70 \%$ [2]. Therefore, the performance of an ipsilateral axillary lymph node dissection (ALND) during the traditional surgical procedures is necessary [3]. While a large number of studies have shown that patients with ALND have some sequelae, since ACOSOG Z0011 study less axillary surgery is being performed, and it is very important to identify the sentinel lymph node (SLN), so not to over treatment with the potential sequelae or undertreatment with potential recurrence $[4,5]$.

The SLN is the first site of mammary lymphatic drainage, and the risk of other lymph node metastasis is extremely low in the presence of SLNs without cancer metastasis. Moreover, patients without SLN metastasis can avoid ALND, which can improve their quality of life without having a significant impact on the long-term survival rate $[6,7]$. Therefore, sentinel lymph node biopsy (SLNB) by "double dye", especially blue-dye method combined with the isotopic tracer method,has become the standard surgical procedure for breast cancer patients with clinically node negative axilla [8]. However, the isotopic tracer method has not yet been applied to clinical work because of its radioactivity in China, so most hospitals adopt the single blue-dye method, which relies heavily on the experience of the surgeon.

Some studies $[9,10]$ have found that breast cancer patients have different lymphatic drainage patterns and number of SLNs, and contrast-enhanced ultrasound (CEUS) represents a feasible method of identifying the diversity of lymphatic drainage patterns and SLNs in breast cancer patients. This is because the UCA can freely enter the lymphatic capillaries across the lymphatic endothelial cell space and then drain to the lymph nodes. The SLN are the first enhanced lymph nodes that are traced along the enhanced lymphatic vessels. Previous studies searching for SLN via CEUS have failed to reach a consensus regarding the injection site and dose of UCA, and the SLN identification rate also remains highly variable. The UCA injection sites in previous studies include cubital vein, intra-tumor, peritumor, and areola injections. In addition, the injection depth includes intradermal, subcutaneous, and intra-gland injections, and the injection dose varies from $0.2 \mathrm{~mL}$ to $2.4 \mathrm{~mL}$.

In this study, by referring to the previous literature on serching for SLN via CEUS, analyzing the influence of different injection sites and dose of UCA on the identification rate, a new injection method of UCA was developed to improve the identification rate of SLN by CEUS.

\section{Methods \\ Patients}

This study was approved by the ethics committee of Sichuan Academy of Medical Sciences \& Sichuan Provincial People's Hospital.

Patients diagnosed with malignant breast tumors by biopsy from the Department of Breast Surgery at Sichuan Provincial People's Hospital were recruited to the present study. According to the SLNB indications mentioned in the guidelines and norms for the diagnosis and treatment of breast cancer by the Chinese anti-cancer association (2017) [8] and the appropriate application of CEUS, the specific inclusion criteria for this study were as follows: (1) patients with a malignant breast tumor; (2) negative for clinical axillary lymph nodes; (3) presence of single or multicentric lesions; (4) any age and sex; (5) plan to undergo a mastectomy and sentinel lymph node biopsy; (6) plan to undergo a breast conserving surgery and sentinel lymph node biopsy; (7) voluntarily accept the examination of CEUS-mediated tracking of SLNs and signing of informed consent. The specific exclusion criteria were: (1) inflammatory breast cancer; (2) severe lung function impairment; (3) allergy to the ultrasonic contrast agent; (4) a history of axillary surgery or radiotherapy; (5) distant metastasis of breast cancer.

\section{Methods}

All of the patients received CEUS the day before or the day of surgery using a Philips iU Elite. The assistant first extracted $5 \mathrm{~mL}$ of normal saline, which was injected into the UCA (59 mg SF6) and was fully mixed prior to the injection. Then, $0.9 \mathrm{~mL}$ normal saline and $0.1 \mathrm{~mL}$ carbon nanoparticles were extracted to prepare the dyes for future injection. Each patient was placed in the supine position, the upper limb of the affected side was lifted up into the same position as the intraoperative position, and the breast and axilla of the affected side was fully exposed. An intradermal injection of $1 \mathrm{~mL}$ UCA at 3 and $6 \mathrm{o}$ 'clock and a subcutaneous injection of $1 \mathrm{~mL}$ UCA at 9 and 12 o 'clock of the mammary areola region was performed following disinfection. The injection site was then massaged gently until the lymphatic vessels began to enhanced to ensure adequate absorption of the 


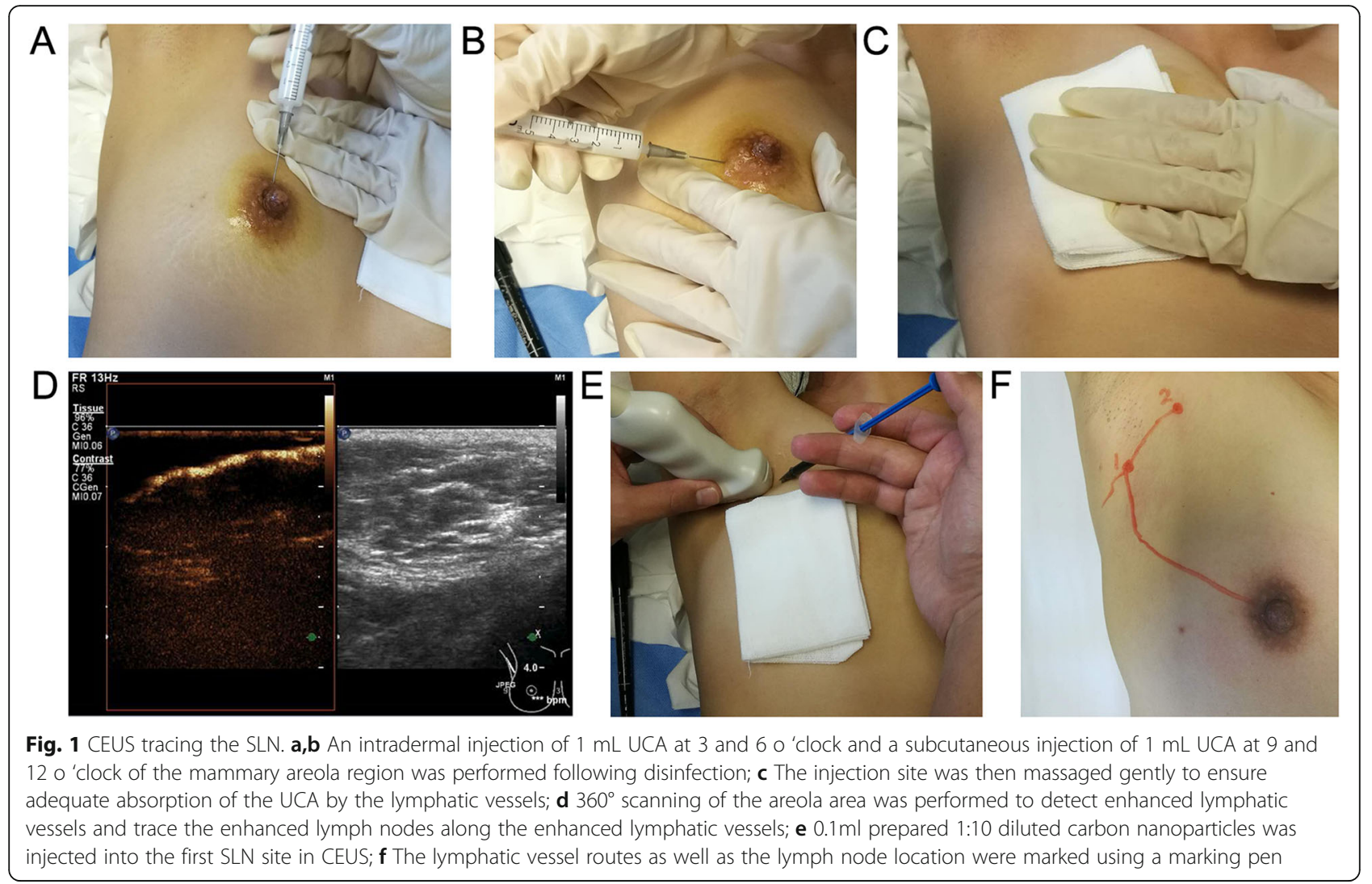

UCA by the lymphatic vessels. A Philips iU Elite was set to contrast-mode, and $360^{\circ}$ scanning of the areola area was performed to detect enhanced lymphatic vessels and trace the enhanced lymph nodes along the enhanced lymphatic vessels with a L9-3 high frequency linear array probe. $0.1 \mathrm{ml}$ prepared 1:10 diluted carbon nanoparticles was injected into the first SLN site in CEUS, and the number of lymph nodes traced by CEUS were recorded and the lymphatic vessel routes as well as the lymph node location were marked using a marking pen (Fig. 1). All

Table 1 The histology of 356 breast cancer patients

\begin{tabular}{lc}
\hline Histology & Number \\
\hline Invasive breast cancer & 275 \\
Ductal carcinoma in situ & 71 \\
Intraductal papillary carcinoma & 16 \\
Mucinous carcinoma & 10 \\
Tubular carcinoma & 2 \\
Adenoid cystic carcinoma & 1 \\
Secretory carcinoma & 1 \\
Solid papillary carcinoma & 4 \\
Malignant phyllodes tumor & 6 \\
Borderline lobe tumor & 4 \\
Total & 390 \\
\hline
\end{tabular}

CEUS examinations were performed by an experienced sonographer who had been engaged in breast ultrasound and intervention ultrasound for more than five years. All of the patients who underwent CEUS received a multipoint injection with MB subcutaneously and intradermally around the areola region 15 min before surgery. At surgery, after incising the skin, the dyed lymph nodes were traced along the dyed lymphatic vessels, and the results were compared with that of CEUS. The SLNs detected by CEUS and MB, as well as the enlarged lymph nodes in adjacent regions were all sent for pathological biopsy.

\section{Statistical analysis}

SPSS17.0 was used for data processing. The measurement data were expressed as $` \pm \mathrm{s}$ if they were normally distributed, and the median (M) and upper and lower quartiles (P25, P75) were used if they were not normally

Table 2 The SLN identification rate by CEUS and MB

\begin{tabular}{lrrrrr}
\hline CEUS & MB & & & Total & \\
\cline { 2 - 3 } & + & - & & \\
\hline+ & 351 & 22 & 373 & \\
- & 8 & 9 & 17 & \\
Total & 359 & 31 & 390 & $*_{P=0.016}$ \\
\hline
\end{tabular}

*Compared with the identification rate of SLN traced by CEUS and MB, a $x^{2}$ test was used, and the difference was statistically significant $(P=0.016)$ 
Table 3 The classification of SLNs according to CEUS and Methylene blue (MB)

\begin{tabular}{lccc}
\hline Classification & Number of cases & Number of positive cases & Number of negative cases \\
\hline${ }^{a} \mathrm{CEUS}^{+} / \mathrm{MBB}^{+}$ & 351 & 61 & 290 \\
$\mathrm{CEUS}^{+} / \mathrm{MB}^{-}$ & 22 & 9 & 13 \\
${ }^{\mathrm{c}} \mathrm{CEUS}{ }^{-} / \mathrm{MB}^{+}$ & 8 & 0 & 8 \\
$\mathrm{CEUS}^{-} / \mathrm{MB}^{-}$ & 9 & 8 & 1 \\
Total & 390 & 78 & 312
\end{tabular}

a enhanced SLNs were observed by CEUS ${ }^{\mathrm{b}}$ Blue-stained SLNs were observed by $\mathrm{MB}^{\mathrm{c}}$ enhanced SLNs were not observed by CEUS ${ }^{\mathrm{d}}$ Blue-stained SLNs were not observed by MB

distributed. The identification rate was defined by the proportion of patients with SLNs identified using either method. The comparison of the SLN identification rate between the two methods was subjected to a $X^{2}$ test. The level of significance was set at $P<0.05$. If the number of lymph nodes detected by the two methods conformed to normal distribution, the paired sample $\mathrm{T}$ test was used for comparison; if not, the paired sample rank sum test was used. The level of significance was set at $P<0.05$.

\section{Results}

A total of 390 breast cancer patients from our breast surgery department from July 2017 to February 2019 were analyzed in the present study. Table 1 presents the histology results. All of the patients were female, aged from 22 to 85 years old, with a median age of 49(43,60). All the lesions were single, with a maximum diameter of $0.5 \mathrm{~cm}-6.0 \mathrm{~cm}$.

Among the 390 cases of breast cancer, enhanced SLNs were observed in 373 patients after the injection of
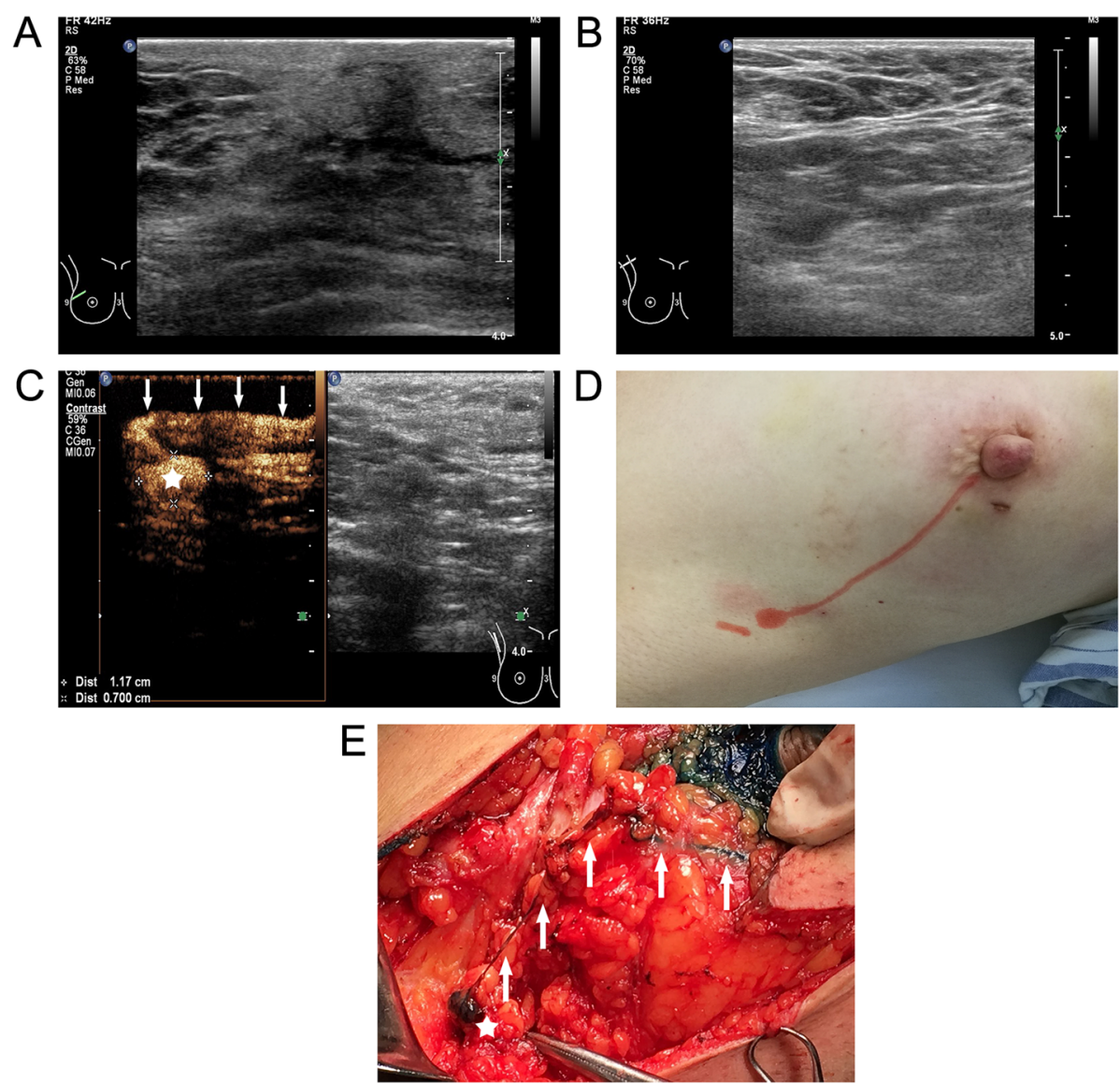

Fig. 2 Case1, 54 year-old woman, Ductal carcinoma in situ. a B-Mode Ultrasonography of right external upper quadrant ductal carcinoma in situ; b B-Mode Ultrasonography of the right axillary; c traced the enhanced SLN (asterisk) along the enhanced lymphatic vessel (arrows) after injecting the UCA; d The body surface projection of enhanced lymphatic vessel and lymph node; eTraced the dyed SLN (asterisk) along the dyed lymphatic vessel (arrows) after injecting the MB before surgery, the lymphatic drainage pattern traced by CEUS was consistent with MB 
UCA, with an identification rate of $95.64 \%(373 / 390)$. And the identification rate of the intraoperative dye method was $92.05 \%$ (359/390), The difference between the two methods was statistically significant $(P=0.016)$. The details of these identification are shown in Tables 2 and 3. And whether the SLNs had metastases are shown in Table 3. (The cases of CEUS vs. MB are shown in Figs. 2 and 3).

A total of 808 enhanced lymph nodes were traced in CEUS, with a median of $2(1,3)$. And 971 blue-stained lymph nodes were traced by intraoperative injection of $M B$, with a median of $2(2,3)$, which could observe significantly more lymph nodes $(P<0.001)$. Please refer to Table 4 for the specific number of lymph nodes.

\section{Discussion}

Grade 1 evidence of evidence-based medicine (EBM) shows that SLNB can safely and effectively replace ALND in patients without SLN metastasis, and the survival rate of SLNB was not worse than that of ALND after receiving standardized adjuvant therapy, dispite one or two SLNs metastasis [11,12]. The key purpose of SLNB is to accurately identify and locate SLNs so as to reduce the potential false negative rate.

Some studies have found that CEUS has the potential to identify SLNs, While these studies have failed to reach a consensus regarding the injection site and dose of UCA. Some of the studies have shown that intra-tumor, peritumor, and areola injections have similar
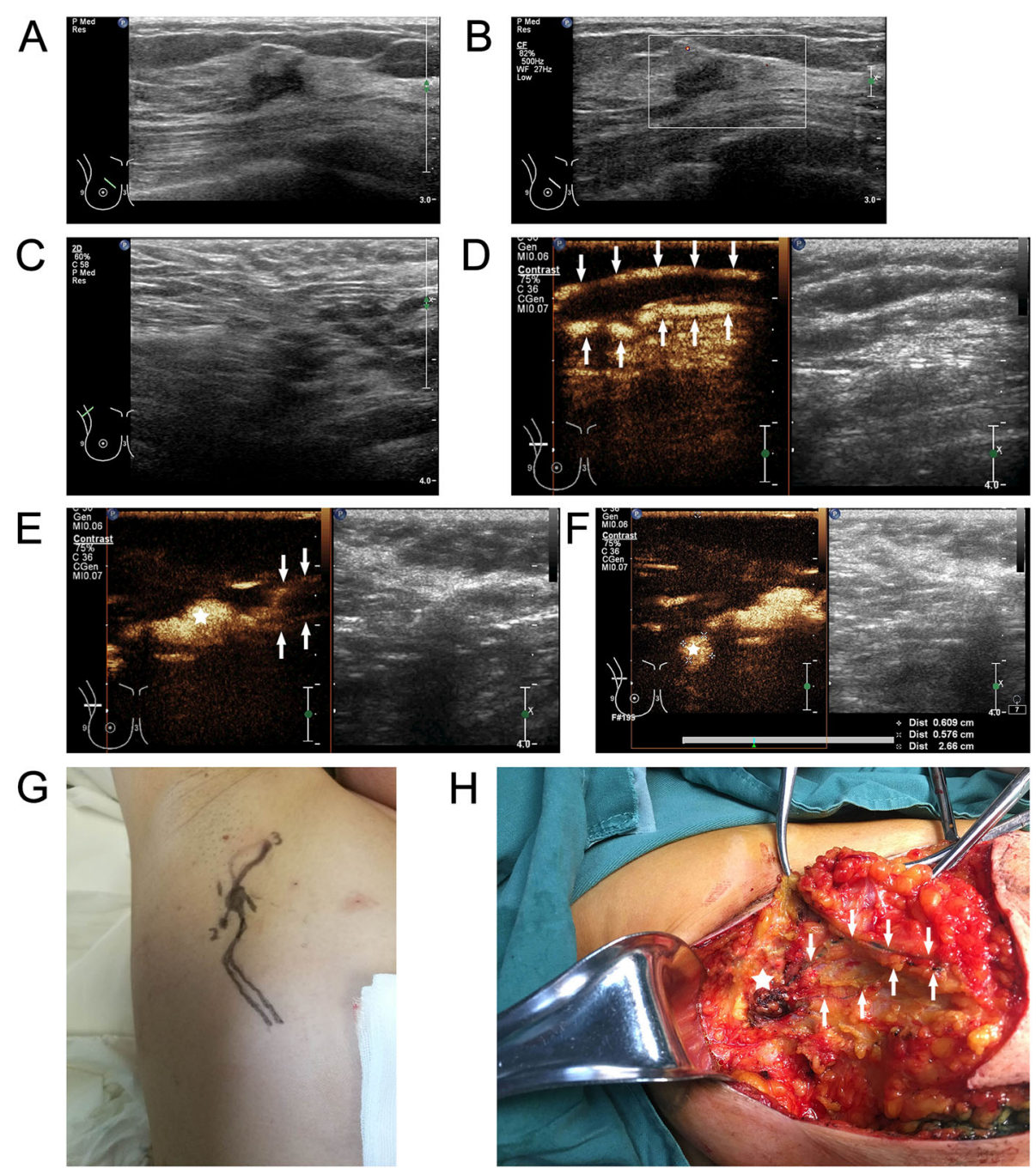

Fig. 3 Case2, 52 year-old woman, Invasive ductal carcinoma.a B-Mode Ultrasonography of right inner upper quadrant invasive ductal carcinoma; b Color-Doppler Ultrasonography of right inner upper quadrant invasive ductal carcinoma; c B-Mode Ultrasonography of the right axillary; dTraced two enhanced lymphatic vessel (arrows) after injecting the UCA; e Both lymphatic vessel (arrows) drain to the same enhanced SLN (asterisk); fUCA can continue to drain to subsequent enhanced lymph node (asterisk); $\mathbf{g}$ The body surface projection of enhanced lymphatic vessel and lymph node; $\mathbf{h}$ Traced two dyed lymphatic vessel (arrows) drain to the same enhanced SLN (asterisk) after injecting the MB before surgery, the lymphatic drainage pattern traced by CEUS was consistent with MB 
Table 4 The number of lymph nodes per patient traced by CEUS and MB

\begin{tabular}{|c|c|c|c|c|}
\hline \multirow{2}{*}{$\begin{array}{l}\text { no. } \\
\text { lymph } \\
\text { nodes }\end{array}$} & \multicolumn{4}{|c|}{ The number and percentage of patients } \\
\hline & CEUS & Percentage (\%) & MB & Percentage (\%) \\
\hline 0 & 17 & 4.4 & 31 & 7.9 \\
\hline 1 & 120 & 30.8 & 26 & 6.7 \\
\hline 2 & 132 & 33.8 & 155 & 39.7 \\
\hline 3 & 71 & 18.2 & 106 & 27.2 \\
\hline 4 & 39 & 10.0 & 43 & 11.0 \\
\hline 5 & 11 & 2.8 & 29 & 7.4 \\
\hline Total & 390 & 100.0 & 390 & 100.0 \\
\hline
\end{tabular}

※ Compared with the number of lymph nodes traced by CEUS and MB, the data did not conform to a normal distribution, and the paired sample rank sum test was used. $P<0.001$, the difference was statistically significant. MB could observe more lymph nodes

identification rate of SLNs [13, 14]. Some other studies and meta-analysis suggested that areola injection has a higher identification rate of SLNs $[15,16]$. Moreover, an intradermal or subcutaneous injection of UCA is associated with a greater identification rate of SLNs compared to an injection into the mammary gland, which is due to the higher density of the superficial lymphatic network. By referring to the previous literature, in this study, an intradermal injection of $1 \mathrm{~mL}$ UCA at 3 and 6 o 'clock, as well as a subcutaneous injection of $1 \mathrm{ml}$ UCA at 9 and $12 \mathrm{o}$ 'clock into the mammary areola region was performed. This method was used to ensure that the lymphatic vessels under the areola region fully absorb the UCA and drain to the sentinel node area, the identification rate of the CEUS method was 95.64\% (373/390).

There were 9 out of 17 patients who did not have enhanced SLN with CEUS, in which interrupted lymphatic vessels and no dyeing of the lymph node was observed using the intraoperative dye method, 8 cases of the pathological results from ALND revealed ALN with cancer metastasis. It is possible that metastatic cancer cells may completely obstruct the lymphatic vessels, resulting in the inability of UCA and MB to drain to the lymph nodes [17]. The dyeing of lymph nodes using the intraoperative dye method and the pathological results were observed to be benign in the other 8 patients. Moreover, each of these patients had large tumors or hematoma following a puncture in the outer upper quadrant, which can compress the lymphatic vessels and affect the absorption and drainage of the UCA. This observation may be related to the different molecular weights of the tracers and physiological mechanisms of lymphatic absorption. It may also be that the lymphatic vessels only absorb a small amount of UCA, resulting in a weak lymphatic enhancement that is overlooked.

There were 351 cases out of the 373 patients in which enhanced SLNs observed by CEUS displayed dyeing of the lymph nodes using the intraoperative dye method. In another 22 cases, interrupted lymphatic vessels and no dyeing of the lymph node was observed with the intraoperative dye method, of which 9 cases displayed lymph node metastasis. Thus, CEUS can identify some SLNs that cannot be detected using the dye method. This is consistent with the findings of Xie et al., [18] in which patients who were not tracked for SLN staining with the intraoperative blue-dye method received CEUS examination before surgery. Thus, these patients received SLNB conditionally, avoiding the deficiency of the single dye method.

Regarding the number of observed lymph nodes, more lymph nodes were observed with MB than CEUS, which is consistent with the results of some previous studies $[2,18-21]$. Since MB has a small molecular weight and lymphatic vessels have good absorbency of $\mathrm{MB}$, when it drains to the SLN after injection, there is partial drainage to subsequent lymph nodes other than the SLNs, causing some of the subsequent lymph nodes to be stained blue during the process of intraoperative detection. However, the dye method requires that all stained

Table 5 Characteristics of included studies

\begin{tabular}{|c|c|c|c|c|c|c|}
\hline $\begin{array}{l}\text { Author, } \\
\text { year }\end{array}$ & $\begin{array}{l}\text { Patients } \\
\text { (n) }\end{array}$ & Injection site & Injection dose & $\begin{array}{l}\text { Identification } \\
\text { rate }\end{array}$ & $\begin{array}{l}\text { Number of } \\
\text { SLNs }\end{array}$ & $\begin{array}{l}\text { Localization } \\
\text { method }\end{array}$ \\
\hline $\begin{array}{l}\text { Liu,2019 } \\
{[24]}\end{array}$ & 75 & $\begin{array}{l}\text { Intradermally inject at 3,6,9,12 o'clock around } \\
\text { the arela }\end{array}$ & $0.5 \mathrm{ml} \times 4$ & $\begin{array}{l}94.67 \% \\
(71 / 75)\end{array}$ & 116 & Skin marked \\
\hline $\begin{array}{l}L i, 2019 \\
{[23]}\end{array}$ & 453 & $\begin{array}{l}\text { Intradermally inject at 3,6,9,12 o'clock around } \\
\text { the arela }\end{array}$ & $0.6 \mathrm{ml} \times 4$ & $\begin{array}{l}98.2 \% \\
(445 / 453)\end{array}$ & 765 & Skin marked \\
\hline $\begin{array}{l}\text { Kenzo, } \\
2019[22]\end{array}$ & 75 & $\begin{array}{l}\text { Intradermally and subdermally inject at 3,6,9,12 } \\
\text { o'clock around the arela }\end{array}$ & $0.25 \mathrm{ml} \times 8$ & $\begin{array}{l}100 \% \\
(100 / 100)\end{array}$ & 92 & Skin marked \\
\hline $\begin{array}{l}\text { Zhong, } \\
2018[19]\end{array}$ & 126 & $\begin{array}{l}\text { Intradermally inject at outer upper quadrant } \\
\text { near the areola }\end{array}$ & $2 \mathrm{ml}$ & $\begin{array}{l}100 \% \\
(126 / 126)\end{array}$ & 164 & FNA \\
\hline $\begin{array}{l}\text { Xie,2015 } \\
{[18]}\end{array}$ & 101 & $\begin{array}{l}\text { Intradermally inject at outer upper quadrant } \\
\text { near the areola }\end{array}$ & $1.5 \mathrm{ml}$ & $\begin{array}{l}97.03 \%(98 / \\
101)\end{array}$ & 115 & Guidewire \\
\hline $\begin{array}{l}\text { Zhao,2018 } \\
{[20]}\end{array}$ & 110 & Intradermally inject at the periareolar area & $\begin{array}{l}0.4 \mathrm{ml} \\
\text { (One or two additional } \\
\text { injections if failed) }\end{array}$ & $\begin{array}{l}96.4 \% \\
(106 / 110)\end{array}$ & 134 & Skin marked \\
\hline
\end{tabular}


lymph nodes to be sent for pathological biopsy, and there is currently no limitation on the number of lymph nodes, which may lead to the removal of too many nonsentinel lymph nodes and loss of SLNB significance. CEUS, on the other hand, is a real-time and dynamic imaging method. With the continuously tracking of enhanced lymph nodes along the enhanced lymphatic vessels immediately after the injection of UCA, some deep or unenhanced subsequent draining lymph nodes were not detected. In some cases, enhanced subsequent lymph node can be observed, but the first site of enhanced lymph nodes and the subsequent lymph nodes can be identified more readily by observing the time of UCA arrival and its connection to the lymphatic vessels.

Previous reports have shown that the identification rate of SLN by CEUS was $70 \% \sim 100 \%$ [21]. The injection site and dose of UCA are inconsistent, and the SLN localization methods are diversified. Table 5 lists some of the literature reports with relatively high identification rate of SLN [18-20, 22-24]. Liu et al. [24] and Zhong et al. [19] had 9 and 8 cases, respectively, of presumed SLNs located by conventional ultrasound and interrupted enhanced lymphatic vessels, which may result in their higher identification rates than they actually were. Li et al. [23] observed that $85.4 \%$ of lymphatic vessels began at upper outer quadrant, $14.6 \%$ lymphatic vessels at other quadrants. Thus, the injection site of UCA should be in four quadrants of the areola to avoid missing enhanced SLNs. In our study, 8 patients failed to observe SLNs by CEUS, which had large tumors or hematoma following a puncture in the outer upper quadrant. And Xie et al. [18] chose the outer edge of the incision as the injection site instead if an incision was performed in upper outer quadrant previously. Multiple injection sites may improve SLN identification rate, but there is no consensus on the optimal injection dose. The body surface location alone was not accurate relatively due to the high activity of mammary gland, SLNs should be marked with more precise methods such as guidewire or staining.

$\mathrm{MB}$ is prone to allergic reactions, local skin flap and adipose tissue necrosis in previous study [21], and one patient developed localized skin necrosis in our study. No patients had adverse reaction to CEUS in this study.In addition, CEUS can display lymphatic vessels and lymph nodes visually. The lymphatic route and SLNs position in the body surface is marked using a marker pen, which can help surgeons to more accurately select the surgical incision, identify lymphatic vessels and SLNs more easily. Moreover, $0.1 \mathrm{ml}$ prepared diluted carbon nanoparticles was injected into the SLNs in our study, to ensure that the black lymph nodes which the surgeon excise were the SLNs that were traced by CEUS.There are some limitations associated with this study. Due to the injection of UCA into the areola area, some patients with pain sensitivity experienced high levels of pain. Additionally, due to the control of domestic nuclides, the patients in this study could not track SLNs via nuclide tracing, and could only be compared using the dye method. Previous literature $[18,20]$ has shown that the identification rate of SLN can be improved by injecting the UCA again or injecting the UCA at the outer edge of the incision in the outer upper quadrant but we didn't do that in our research, that's something we can improve on.

\section{Conclusions}

Intradermal and subcutaneous injections of UCA in the mammary areola region may have clinical application value for the identification and localization of SLNs in breast cancer patients. And it can identify some SLNs which cannot be identified using the dye method, which can be used as a new tracer of SLNB and complement other staining methods to improve the success rate.

\section{Abbreviations \\ ALN: Axillary lymph node; ALND: Axillary lymph node dissection; \\ CEUS: Contrast-enhanced ultrasound; EBM: Evidence-based medicine; MB: Methylene blue; SLN: Sentinel lymph node; SLNB: Sentinel lymph node biopsy; UCA: Ultrasonic contrast agent}

\section{Acknowledgements}

We thank International Science Editing () for editing this manuscript.

\section{Authors' contributions}

$J L, J P L, C H W$, and $J$ conceived and coordinated the study, designed, and analyzed the experiments. JL, JC, HW, WYD and LTF performed the experiments. JL and LTF carried out the data collection, data analysis, and wrote the paper. QZ and QC revised the paper. All authors reviewed the results and approved the final version of the manuscript.

\section{Funding}

Not applicable.

\section{Availability of data and materials}

The datasets used and/or analyzed during the current study are available from the corresponding author on reasonable request.

\section{Declarations}

\section{Ethics approval and consent to participate}

This study was approved by the ethics committee of Sichuan Academy of Medical Sciences \& Sichuan Provincial People's Hospital. Written informed consent was obtained from all individual participants included in the study.

Consent for publication

Not applicable.

\section{Competing interests}

The authors declare that they have no conflict of interest.

\section{Author details}

'Department of Ultrasound, Sichuan Provincial People's Hospital, University of Electronic Science and Technology of China, 32\# W.Sec 2,1st Ring Rd, 610072 Chengdu, China. ${ }^{2}$ Department of Breast Surgery, Sichuan Provincial People's Hospital, University of Electronic Science and Technology of China, 610072 Chengdu, China. 
Received: 28 August 2020 Accepted: 4 March 2021

Published online: 12 March 2021

\section{References}

1. Shi J, Liang D, Li DJ. Epidemiological status of global female breast cancer. China Cancer. 2017;26:683-90.

2. Gkegkes ID, lavazzo C. Contrast enhanced ultrasound (CEU) using microbubbles for sentinel lymph node biopsy in breast cancer: a systematic review. Acta Chir Belg. 2015;115:212-8.

3. Valero MG, Golshan M. Management of the axilla in early breast cancer. Cancer Treat Res. 2018;173:39-52.

4. Julian T, Anderson S, Krag D. Abstract S2-05: 10-yr follow-up results of occult detected sentinel node disease: NSABP B-32, a randomized phase III clinical trial to compare sentinel node resection (SNR) to conventional axillary dissection (AD) in clinically node-negative breast cancer patients. Cancer Res. 2013;73:S2-05-S2

5. Giuliano AE, Hunt KK, Ballman KV, Beitsch PD, Whitworth PW, Blumencranz PW, et al. Axillary dissection vs no axillary dissection in women with invasive breast cancer and sentinel node metastasis: a randomized clinical trial. JAMA. 2011:305:569-75.

6. Lyman GH, Somerfield MR, Bosserman LD, Perkins CL, Weaver DL, Giuliano AE. Sentinel lymph node biopsy for patients with early-stage breast cancer: American Society of Clinical Oncology clinical practice guideline update. J Clin Oncol. 2017;35:561-4.

7. Krag DN, Anderson SJ, Julian TB, Brown AM, Harlow SP, Costantino JP, et al. Sentinel-lymph-node resection compared with conventional axillary-lymphnode dissection in clinically node-negative patients with breast cancer: overall survival findings from the NSABP B-32 randomised phase 3 trial. Lancet Oncol. 2010;11:927-33.

8. Peek MC, Charalampoudis P, Anninga B, Baker R, Douek M. Blue dye for identification of sentinel nodes in breast cancer and malignant melanoma: a systematic review and meta-analysis. Future Oncol. 2017;13:455-67.

9. Wang M, Zhou W, Zhao Y, Xia T, Zha X, Ding Q, et al. A novel finding of sentinel lymphatic channels in early stage breast cancer patients: which may influence detection rate and false-negative rate of sentinel lymph node biopsy. PLoS One. 2012;7:e51226.

10. Wang Y, Zhou W, Li C, Gong H, Li C, Yang N, et al. Variation of sentinel lymphatic channels (SLCS) and sentinel lymph nodes (SLNs) assessed by contrast-enhanced ultrasound (CEUS) in breast cancer patients. World J Surg Oncol. 2017;15:127.

11. Giuliano AE, McCall L, Beitsch P, Whitworth PW, Blumencranz P, Leitch AM, et al. Locoregional recurrence after sentinel lymph node dissection with or without axillary dissection in patients with sentinel lymph node metastases: the American College of Surgeons Oncology Group Z0011 randomized trial. Ann Surg. 2010;252:426-32 discussion 32 - 3.

12. Chinese Anti-Cancer Association, Committee of Breast Cancer Society. Guidelines and norms for the diagnosis and treatment of breast cancer of the Chinese anti-cancer association. China Oncology. 2017:27:695-759.

13. Guo KP, Zhong FY, Cai ZX. Comparative study of injection location of sentinel lymph nodes in breast cancer with under areola and injection around the tumor. J Clin Exper Med. 2018;17:2450-3.

14. Wu Y, Mi C, Wen W. Comparison of peritumoral and subareola ultrasound contrast agents injection in detection of breast cancer sentinel lymph nodes. Chin J Ultrasound Med. 2014;30:210-2.

15. Yang SL, Tang KQ, Tao JJ. Clinical study of different parts of ultrasound contrast agent in the diagnosis of sentinel lymph node in breast cancer. J Clin Exper Med. 2016;15:1773-6.

16. Fu MG, Liu S, Guo L. Subareolar versus peritumoral tracer injection for location of sentinel lymph node in breast carcinoma: a Meta-analysis. Chin J Gen Surg. 2015;24:663-8

17. Shen E, Li C, Wang C. The clinical value of preoperative axillary contrastenhanced ultrasonography combined with core-needle biopsy to the assessment of axillary lymph node status in breast cancer. Chin J Exper Surg. 2017;34:285-7.

18. Xie F, Zhang D, Cheng L, Yu L, Yang L, Tong F, et al. Intradermal microbubbles and contrast-enhanced ultrasound (CEUS) is a feasible approach for sentinel lymph node identification in early-stage breast cancer. World J Surg Oncol. 2015:13:319.

19. Zhong J, Sun DS, Wei W, Liu X, Liu J, Wu X, et al. Contrast-enhanced ultrasound-guided fine-needle aspiration for sentinel lymph node biopsy in early-stage breast cancer. Ultrasound Med Biol. 2018;44:1371-8.
20. Zhao J, Zhang J, Zhu QL, Jiang YX, Sun Q, Zhou YD, et al. The value of contrast-enhanced ultrasound for sentinel lymph node identification and characterisation in pre-operative breast cancer patients: A prospective study. Eur Radiol. 2018;28:1654-61.

21. Cui Q, Dai L, Li J, Xue J. Accuracy of CEUS-guided sentinel lymph node biopsy in early-stage breast cancer: a study review and meta-analysis. World J Surg Oncol. 2020;18(1):112

22. Shimazu K, Miyake T, Tanei T, Naoi Y, Shimoda M, Kagara N, et al. Real-time visualization of lymphatic flow to sentinel lymph nodes by contrastenhanced ultrasonography with sonazoid in patients with breast cancer. Ultrasound Med Biol. 2019:45:2634-40.

23. Li J, Lu M, Cheng X, Hu Z, Li H, Wang H, et al. How pre-operative sentinel lymph node contrast-enhanced ultrasound helps intra-operative sentinel lymph node biopsy in breast cancer: initial experience. Ultrasound Med Biol. 2019:45:1865-73.

24. Liu J, Liu X, He J, Gou B, Luo Y, Deng S, et al. Percutaneous contrastenhanced ultrasound for localization and diagnosis of sentinel lymph node in early breast cancer. Sci Rep. 2019;9:13545.

\section{Publisher's Note}

Springer Nature remains neutral with regard to jurisdictional claims in published maps and institutional affiliations.
Ready to submit your research? Choose BMC and benefit from:

- fast, convenient online submission

- thorough peer review by experienced researchers in your field

- rapid publication on acceptance

- support for research data, including large and complex data types

- gold Open Access which fosters wider collaboration and increased citations

- maximum visibility for your research: over $100 \mathrm{M}$ website views per year

At $\mathrm{BMC}$, research is always in progress.

Learn more biomedcentral.com/submissions 